\title{
ASYMPTOTIC BEHAVIOR OF FRACTIONAL ORDER RIEMANN-LIOUVILLE VOLTERRA-STIELTJES INTEGRAL EQUATIONS
}

\author{
SAÏD ABBAS, MOUFFAK BENCHOHRA, BOUALEM A. SLIMANI \\ AND JUAN J. TRUJILLO \\ Communicated by William McLean
}

\begin{abstract}
In this paper, we present some results concerning the existence and global asymptotic stability of solutions for a functional integral equation of fractional order. We use Schauder's fixed point theorem for the existence of solutions, and we prove that all these solutions are globally asymptotically stable.
\end{abstract}

1. Introduction. Integral equations are a useful mathematical tool in both pure and applied analysis. This is particularly true of problems in mechanical vibrations and the related fields of engineering and mathematical physics. We can find numerous applications of differential and integral equations of fractional order in viscoelasticity, electrochemistry, control, porous media, electromagnetism, etc., $[\mathbf{1 0}, \mathbf{1 7}, \mathbf{2 0}, \mathbf{2 2}, \mathbf{2 4}]$. There has been a significant development in ordinary and partial fractional differential equations in recent years; see the monographs of Abbas, et al., [5, 6], Kilbas, et al., [18], Lakshmikantham [19], Miller and Ross [20], Podlubny [22] and Zhou [25], and the papers by Abbas, et al., $[\mathbf{1}, \mathbf{2}, \mathbf{4}, \mathbf{8}]$, Banaś, et al., [11, 12, 13], Darwish, et al., [15], and the references therein.

In [3], Abbas and Benchohra studied the existence and stability of solutions of the following nonlinear quadratic Volterra integral equation

2010 AMS Mathematics subject classification. Primary 26A33, 45G05, 45M10.

Keywords and phrases. Volterra-Stieltjes integral equation, left-sided mixed Riemann-Liouville integral of fractional order, solution, global asymptotic stability, fixed point.

This work was partially funded by the Government of Spain and FEDER (grant No. 163 MTM2013-41704-P).

Received by the editors on January 5, 2015, and in revised form on April 4, 2015. DOI:10.1216/JIE-2015-27-3-311 
of Riemann-Liouville fractional order,

$$
u(t)=f(t, u(\alpha(t)))+\frac{1}{\Gamma(r)} \int_{0}^{\beta(t)}(\beta(t)-s)^{r-1} g(t, s, u(\gamma(s))) d s
$$

for $t \in \mathbb{R}_{+}$, where $\alpha, \beta, \gamma: \mathbb{R}_{+} \rightarrow \mathbb{R}_{+}, f: \mathbb{R}_{+} \times \mathbb{R} \rightarrow \mathbb{R}$ and $g: \mathbb{R}_{+} \times \mathbb{R}_{+} \times \mathbb{R} \rightarrow \mathbb{R}$ are continuous functions, $\mathbb{R}_{+}=[0, \infty), r \in(0, \infty)$ and $\Gamma$ is the (Euler) gamma function defined by

$$
\Gamma(\xi)=\int_{0}^{\infty} t^{\xi-1} e^{-t} d t, \quad \xi>0 .
$$

In [7], Abbas, et al., considered the existence of solutions of fractional order, Riemann-Liouville, Volterra-Stieltjes quadratic integral equations of the form

$$
\begin{aligned}
u(t, x)= & \mu(t, x) \\
& +\frac{f(t, x, u(t, x))}{\Gamma(r)} \int_{0}^{t}(t-s)^{r-1} h(t, x, s, u(s, x)) d_{s} g(t, s)
\end{aligned}
$$

for $(t, x) \in J:=[0, a] \times[0, b]$, where $a, b>0, r \in(0, \infty) ; \mu: J \rightarrow \mathbb{R}$, $g: \mathbb{R}_{+} \times \mathbb{R}_{+} \rightarrow \mathbb{R}, f: J \times \mathbb{R} \rightarrow \mathbb{R}$ and $h: J_{1} \times \mathbb{R} \rightarrow \mathbb{R}$ are given continuous functions, and $J_{1}=\{(t, x, s) \in J \times[0, a]: s \leq t\}$.

Motivated by the above papers, this paper deals with the existence of solutions to the following more general nonlinear functional equation

$$
u(t)=N(u)(t):=f(t, u(\alpha(t)), Q(u)(t)) \text { for } t \in \mathbb{R}_{+},
$$

in which $Q$ is the fractional order Riemann-Liouville, Volterra-Stieltjes integral operator

$$
Q(u)(t)=\frac{1}{\Gamma(r)} \int_{0}^{\beta(t)}(\beta(t)-s)^{r-1} h(t, s, u(\gamma(s))) d_{s} g(t, s),
$$

and where $r>0 ; \alpha, \beta: \mathbb{R}_{+} \rightarrow \mathbb{R}_{+}$are given uniformly continuous functions; $\lim _{t \rightarrow \infty} \alpha(t)=\infty ; \gamma: \mathbb{R}_{+} \rightarrow \mathbb{R}_{+}, f: \mathbb{R}_{+} \times \mathbb{R} \times \mathbb{R} \rightarrow \mathbb{R}$, $g: \mathbb{R}_{+} \times \mathbb{R}_{+} \rightarrow \mathbb{R}$ and $h: J_{1} \times \mathbb{R} \rightarrow \mathbb{R}$ are continuous functions; and $J_{1}:=\left\{(t, s) \in \mathbb{R}_{+}^{2}: s \leq \beta(t)\right\}$. We prove the existence of solutions of equation (1.1) by using Schauder's fixed point theorem, and we obtain some results about the global asymptotic stability of solutions. An example illustrating the main result is presented in the last section. 
2. Preliminaries. In this section, we introduce notation, definitions and preliminary facts which are used throughout this paper. For $b>0$, let $L^{1}([0, b])$ denote the space of Lebesgue-integrable functions $u:[0, b] \rightarrow \mathbb{R}$ with the norm

$$
\|u\|_{1}=\int_{0}^{b}|u(t)| d t
$$

By $\mathrm{BC}:=\mathrm{BC}\left(\mathbb{R}_{+}\right)$, we denote the Banach space of all bounded and continuous functions from $\mathbb{R}_{+}$into $\mathbb{R}$ equipped with the standard norm

$$
\|u\|_{\mathrm{BC}}=\sup _{t \in \mathbb{R}_{+}}|u(t)|
$$

For $u_{0} \in \mathrm{BC}$ and $\eta \in(0, \infty)$, we denote by $B\left(u_{0}, \eta\right)$ the closed ball in $\mathrm{BC}$ centered at $u_{0}$ with radius $\eta$.

Definition 2.1 ([18]). Let $r>0$. For $u \in L^{1}([0, b])$, the expression

$$
\left(I_{0}^{r} u\right)(t)=\frac{1}{\Gamma(r)} \int_{0}^{t}(t-s)^{r-1} u(s) d s, \quad t \in \mathbb{R}_{+},
$$

is called the left-sided Riemann-Liouville fractional integral of order $r$.

In particular, $\left(I_{0}^{0} u\right)(t)=u(t)$ and $\left(I_{0}^{1} u\right)(t)=\int_{0}^{t} u(s) d s$ for almost all $t \in[0, b]$. Note that $I_{0}^{r} u$ exists for all $r>0$ when $u \in L^{1}([0, b])$, and that $\left(I_{0}^{r} u\right) \in C([0, b])$ when $u \in C([0, b])$.

Example 2.2. Let $\omega, r \in(0, \infty)$. Then

$$
I_{0}^{r} \frac{t^{\omega}}{\Gamma(1+\omega)}=\frac{t^{\omega+r}}{\Gamma(1+\omega+r)} \quad \text { for } t \in[0, b] .
$$

If $u$ is a real function defined on the interval $[a, b]$, then the symbol $\bigvee_{a}^{b} u$ denotes the variation of $u$ on $[a, b]$. We say that $u$ is of bounded variation on the interval $[a, b]$ whenever $\bigvee_{a}^{b} u$ is finite. For a function $w:[a, b] \times[c, b] \rightarrow \mathbb{R}$, the symbol $\bigvee_{t=p}^{q} w(t, s)$ indicates the variation of the function $t \mapsto w(t, s)$ on the interval $[p, q] \subset[a, b]$, where $s$ is arbitrarily fixed in $[c, d]$. In the same way, we define $\bigvee_{s=p}^{q} w(t, s)$. For the properties of functions of bounded variation we refer to $[\mathbf{9}, \mathbf{2 1}]$. 
If $u$ and $\varphi$ are two real functions defined on the interval $[a, b]$, then under some conditions (see $[\mathbf{9}, \mathbf{2 1}]$ ) we can define the Stieltjes integral (in the Riemann-Stieltjes sense) of the function $u$ with respect to $\varphi$,

$$
\int_{a}^{b} u(t) d \varphi(t)
$$

In this case we say that $u$ is Stieltjes integrable on $[a, b]$ with respect to $\varphi$. Several conditions are known guaranteeing Stieltjes integrability $[\mathbf{9}, \mathbf{2 1}, \mathbf{2 3}]$. One of the most frequently used requires that $u$ be continuous and $\varphi$ be of bounded variation on $[a, b]$.

In what follows, we will use a few properties of the Stieltjes integral contained in the lemmas given below.

Lemma 2.3 ([9]). If $u$ is Stieltjes integrable on the interval $[a, b]$ with respect to a function $\varphi$ of bounded variation, then

$$
\left|\int_{a}^{b} u(t) d \varphi(t)\right| \leq \int_{a}^{b}|u(t)| d\left(\bigvee_{a}^{t} \varphi\right)
$$

Lemma 2.4 ([9]). Let $u, v$ be Stieltjes integrable functions on the interval $[a, b]$ with respect to a nondecreasing function $\varphi$ such that $u(t) \leq v(t)$ for $t \in[a, b]$. Then

$$
\int_{a}^{b} u(t) d \varphi(t) \leq \int_{a}^{b} v(t) d \varphi(t) .
$$

In the sequel, we will also consider Stieltjes integrals of the form

$$
\int_{a}^{b} u(t) d_{s} g(t, s)
$$

and Riemann-Liouville Stieltjes integrals of fractional order of the form

$$
\frac{1}{\Gamma(r)} \int_{0}^{t}(t-s)^{r-1} u(s) d_{s} g(t, s),
$$

where $g: \mathbb{R}_{+} \times \mathbb{R}_{+} \rightarrow \mathbb{R}, r \in(0, \infty)$ and the symbol $d_{s}$ indicates the integration with respect to $s$. 
Let $\emptyset \neq \Omega \subset \mathrm{BC}$, let $G: \Omega \rightarrow \Omega$, and consider the solutions of equation

$$
(G u)(t)=u(t) .
$$

We introduce the following concept of attractivity of such solutions.

Definition 2.5. Solutions of equation (2.1) are locally attractive if there exists a ball $B\left(u_{0}, \eta\right)$ in the space BC such that, for arbitrary solutions $v=v(t)$ and $w=w(t)$ of equation (2.1) belonging to $B\left(u_{0}, \eta\right) \cap \Omega$, we have

$$
\lim _{t \rightarrow \infty}(v(t)-w(t))=0 .
$$

When the limit $(2.2)$ is uniform with respect to $B\left(u_{0}, \eta\right) \cap \Omega$, solutions of equation (2.1) are said to be uniformly locally attractive (or equivalently, the solutions are locally asymptotically stable).

Definition 2.6. The solution $v=v(t)$ of equation (2.1) is said to be globally attractive if (2.2) holds for each solution $w=w(t)$ of $(2.1)$. If condition (2.2) is satisfied uniformly with respect to the set $\Omega$, solutions of equation (2.1) are said to be globally asymptotically stable (or uniformly globally attractive).

Lemma 2.7 ([14], page 62). Let $D \subset \mathrm{BC}$. Then $D$ is relatively compact in $B C$ if the following conditions hold:

(a) $D$ is uniformly bounded in $B C$;

(b) the functions belonging to $D$ are almost equicontinuous on $\mathbb{R}_{+}$, that is, equicontinuous on every compact subset of $\mathbb{R}_{+}$;

(c) the functions from $D$ are equiconvergent, that is, given $\epsilon>0$, there corresponds $T(\epsilon)>0$ such that $\left|u(t)-\lim _{t \rightarrow \infty} u(t)\right|<\epsilon$ for any $t \geq T(\epsilon)$ and $u \in D$.

3. Main results. In this section, we are concerned with the existence and the global asymptotic stability of solutions for the equation (1.1). Let us start by defining exactly what we mean by a solution.

Definition 3.1. A solution of equation (1.1) is any function $u \in \mathrm{BC}$ such that $u$ satisfies equation (1.1) on $\mathbb{R}_{+}$. 
The following hypotheses will be used in the sequel.

$\left(H_{1}\right)$ There exist constants $0<M<1$ and $L>0$ such that

$$
\left|f\left(t, u_{1}, v_{1}\right)-f\left(t, u_{2}, v_{2}\right)\right| \leq \frac{M\left|u_{1}-u_{2}\right|+L\left|v_{1}-v_{2}\right|}{1+\alpha(t)+\left|u_{1}-u_{2}\right|}
$$

for $t \in \mathbb{R}_{+}$and for $u_{1}, v_{1}, u_{2}, v_{2} \in \mathbb{R}$.

$\left(H_{2}\right)$ The function $t \mapsto f(t, 0,0)$ is bounded on $\mathbb{R}_{+}$with $f^{*}=$ $\sup _{t \in \mathbb{R}_{+}} f(t, 0,0)$ and $\lim _{t \rightarrow \infty}|f(t, 0,0)|=0$.

$\left(H_{3}\right)$ For all $t_{1}$ and $t_{2} \in \mathbb{R}_{+}$such that $t_{1}<t_{2}$, the function $s \mapsto$ $g\left(t_{2}, s\right)-g\left(t_{1}, s\right)$ is nondecreasing on $\mathbb{R}_{+}$.

$\left(H_{4}\right)$ The function $s \mapsto g(0, s)$ is nondecreasing on $\mathbb{R}_{+}$.

$\left(H_{5}\right)$ The functions $s \mapsto g(t, s)$ and $t \mapsto g(t, s)$ are continuous on $\mathbb{R}_{+}$ for each fixed $t \in \mathbb{R}_{+}$or $s \in \mathbb{R}_{+}$, respectively.

$\left(H_{6}\right)$ The function $t \mapsto \bigvee_{0}^{s} g(t, s)$ is continuous on $\mathbb{R}_{+}$for each $s \in \mathbb{R}_{+}$.

$\left(H_{7}\right)$ There exists a continuous function $p: J_{1} \rightarrow \mathbb{R}_{+}$such that $|h(t, s, u)| \leq \frac{p(t, s)}{1+\alpha(t)+|u|} \quad$ for $(t, s) \in J_{1}$ and $u \in \mathbb{R}$

and, moreover,

$$
\lim _{t \rightarrow \infty} \int_{0}^{\beta(t)}(\beta(t)-s)^{r-1} p(t, s) d_{s} g(t, s)=0 .
$$

Remark 3.2. Set $q^{*}:=\sup _{t \in \mathbb{R}_{+}} q(t)$ where

$$
q(t)=\frac{1}{\Gamma(r)} \int_{0}^{\beta(t)}(\beta(t)-s)^{r-1} p(t, s) d_{s}\left(\bigvee_{k=0}^{s} g(t, k)\right)
$$

From hypothesis $\left(H_{7}\right)$, we infer that $q^{*}$ is finite, $\lim _{t \rightarrow \infty} q(t)=0$, and (3.1)

$$
|(Q u)(t)| \leq \frac{1}{\Gamma(r)} \int_{0}^{\beta(t)} \frac{(\beta(t)-s)^{r-1} p(t, s)}{1+\alpha(t)+|u(\gamma(t))|} d_{s}\left(\bigvee_{0}^{s} g(t, \cdot)\right) \leq q(t)
$$

Theorem 3.3. Assume that hypotheses $\left(H_{1}\right)-\left(H_{7}\right)$ hold. Then equation (1.1) has at least one solution in the space BC. Moreover, solutions of equation (1.1) are globally asymptotically stable. 
Proof. Recall the operator $N$ defined by (1.1) and (1.2). It is clear that the map $t \mapsto(N u)(t)$ is continuous on $\mathbb{R}_{+}$. Now we prove that $N(u) \in \mathrm{BC}$ for any $u \in \mathrm{BC}$. For arbitrarily fixed $t \in \mathbb{R}_{+}$, assumptions $\left(H_{1}\right)$ and $\left(H_{7}\right)$, together with $(3.1)$, imply that

$$
\begin{aligned}
|(N u)(t)| & =|f(t, 0,0)|+|f(t, u(\alpha(t)),(Q u)(t))-f(t, 0,0)| \\
& \leq|f(t, 0,0)|+\frac{M|u(\alpha(t))|+L|(Q u)(t)|}{1+\alpha(t)+|u(\alpha(t))|} \\
& \leq|f(t, 0,0)|+M+L q(t) \leq f^{*}+M+q^{*},
\end{aligned}
$$

SO

$$
\|N(u)\|_{\mathrm{BC}} \leq f^{*}+M+L q^{*}
$$

Hence, $N(u) \in$ BC. Equation (3.3) yields that $N$ transforms the ball $B_{\eta}:=B(0, \eta)$ into itself, where $\eta=f^{*}+M+L q^{*}$. We shall show that $N: B_{\eta} \rightarrow B_{\eta}$ satisfies the assumptions of Schauder's fixed point theorem [16]. The proof will be given in several steps.

Step 1. $N$ is continuous. Let $\epsilon>0$. By assumption $\left(H_{7}\right)$, we can choose $T>0$ such that $q(t)<\epsilon /(2 L)$ for all $(t, s) \in J_{1}$ such that $t>T$. Let $\left\{u_{n}\right\}_{n \in \mathbb{N}}$ be a sequence such that $u_{n} \rightarrow u$ in $B_{\eta}$. Then, for each $t \in \mathbb{R}_{+}$, we have

$$
\begin{aligned}
\mid\left(N u_{n}\right)(t) & -(N u)(t) \mid \\
& =\left|f\left(t, u_{n}(\alpha(t)),\left(Q u_{n}\right)(t)\right)-f(t, u(\alpha(t)),(Q u)(t))\right| \\
& \leq \frac{M\left|u_{n}(\alpha(t))-u(\alpha(t))\right|+L\left|\left(Q u_{n}\right)(t)-(Q u)(t)\right|}{1+\alpha(t)+\mid u_{n}(\alpha(t)-u(\alpha(t)) \mid} \\
& \leq M\left\|u_{n}-u\right\|_{\mathrm{BC}}+L\left|\left(Q u_{n}\right)(t)-(Q u)(t)\right|,
\end{aligned}
$$

and $\left|\left(Q u_{n}\right)(t)-(Q u)(t)\right|$ is bounded by

$$
\int_{0}^{\beta(t)}(\beta(t)-s)^{r-1}\left|h\left(t, s, u_{n}(\gamma(s))\right)-h(t, s, u(\gamma(s)))\right| d_{s}\left(\bigvee_{0}^{s} g(t, \cdot)\right)
$$

These estimates show that, because $h$ is continuous and $g$ has bounded variation,

$$
\max _{0 \leq t \leq T}\left\|N\left(u_{n}\right)(t)-N(u)(t)\right\| \longrightarrow 0 \quad \text { as } n \rightarrow \infty
$$


and by (3.1),

$$
\left|\left(Q u_{n}\right)(t)-(Q u)(t)\right| \leq\left|\left(Q u_{n}\right)(t)\right|+|(Q u)(t)| \leq 2 q(t),
$$

so

$$
\sup _{t>T}\left\|N\left(u_{n}\right)(t)-N(u)(t)\right\| \leq M\left\|u_{n}-u\right\|_{\mathrm{BC}}+\epsilon .
$$

It follows that $\left\|N\left(u_{n}\right)-N(u)\right\|_{B C} \rightarrow 0$ as $n \rightarrow \infty$.

Step 2. $N\left(B_{\eta}\right)$ is uniformly bounded. This claim is clear since $N\left(B_{\eta}\right) \subseteq B_{\eta}$ and $B_{\eta}$ is bounded.

Step 3. $N\left(B_{\eta}\right)$ is equicontinuous on every compact interval $[0, a] \subseteq \mathbb{R}_{+}(a>0)$. Let $t_{1}$ and $t_{2} \in[0, a]$ with $t_{1}<t_{2}$, let $u \in B_{\eta}$ and, without loss of generality, assume that $\beta\left(t_{1}\right) \leq \beta\left(t_{2}\right)$. We define

$$
\begin{aligned}
& I_{1}=\left|f\left(t_{2}, u\left(\alpha\left(t_{2}\right)\right),(Q u)\left(t_{2}\right)\right)-f\left(t_{2}, u\left(\alpha\left(t_{1}\right)\right),(Q u)\left(t_{1}\right)\right)\right|, \\
& I_{2}=\left|f\left(t_{2}, u\left(\alpha\left(t_{1}\right)\right),(Q u)\left(t_{1}\right)\right)-f\left(t_{1}, u\left(\alpha\left(t_{1}\right)\right),(Q u)\left(t_{1}\right)\right)\right|,
\end{aligned}
$$

so that

$$
\left|(N u)\left(t_{2}\right)-(N u)\left(t_{1}\right)\right| \leq I_{1}+I_{2} .
$$

By assumption $\left(H_{1}\right)$,

$$
\begin{aligned}
I_{1} & \leq \frac{M\left|u\left(\alpha\left(t_{2}\right)\right)-u\left(\alpha\left(t_{1}\right)\right)\right|+L\left|(Q u)\left(t_{2}\right)-(Q u)\left(t_{1}\right)\right|}{1+\alpha(t)+\left|u\left(\alpha\left(t_{2}\right)\right)-u\left(\alpha\left(t_{1}\right)\right)\right|} \\
& \leq M\left|u\left(\alpha\left(t_{2}\right)\right)-u\left(\alpha\left(t_{1}\right)\right)\right|+\frac{M}{\Gamma(r)}\left(I_{11}+I_{12}+I_{13}\right),
\end{aligned}
$$

for

$$
\begin{aligned}
I_{11}= & \int_{0}^{\beta\left(t_{2}\right)}\left(\beta\left(t_{2}\right)-s\right)^{r-1} h\left(t_{2}, s, u(\gamma(s))\right) d_{s} g\left(t_{2}, s\right) \\
& \left.-\int_{0}^{\beta\left(t_{2}\right)}\left(\beta\left(t_{2}\right)-s\right)^{r-1} h\left(t_{1}, s, u(\gamma(s))\right)\right] d_{s} g\left(t_{2}, s\right), \\
I_{12}= & \int_{0}^{\beta\left(t_{2}\right)}\left(\beta\left(t_{2}\right)-s\right)^{r-1} h\left(t_{1}, s, u(\gamma(s))\right) d_{s} g\left(t_{2}, s\right) \\
& -\int_{0}^{\beta\left(t_{1}\right)}\left(\beta\left(t_{2}\right)-s\right)^{r-1} h\left(t_{1}, s, u(\gamma(s))\right) d_{s} g\left(t_{1}, s\right), \\
I_{13}= & \int_{0}^{\beta\left(t_{1}\right)}\left(\beta\left(t_{2}\right)-s\right)^{r-1} h\left(t_{1}, s, u(\gamma(s))\right) d_{s} g\left(t_{1}, s\right)
\end{aligned}
$$




$$
-\int_{0}^{\beta\left(t_{1}\right)}\left(\beta\left(t_{1}\right)-s\right)^{r-1} h\left(t_{1}, s, u(\gamma(s))\right) d_{s} g\left(t_{1}, s\right) .
$$

Using the estimates

$$
\begin{aligned}
\left|I_{11}\right| \leq & \int_{0}^{\beta\left(t_{2}\right)}\left(\beta\left(t_{2}\right)-s\right)^{r-1} \\
& \left|h\left(t_{2}, s, u(\gamma(s))\right)-h\left(t_{1}, s, u(\gamma(s))\right)\right| d_{s}\left(\bigvee_{0}^{s} g\left(t_{2}, \cdot\right)\right) \\
\left|I_{12}\right| \leq & \int_{\beta\left(t_{1}\right)}^{\beta\left(t_{2}\right)}\left(\beta\left(t_{2}\right)-s\right)^{r-1} p\left(t_{1}, s\right) d_{s}\left(\bigvee_{0}^{s} g\left(t_{2}, \cdot\right)\right) \\
& +\int_{0}^{\beta\left(t_{1}\right)}\left(\beta\left(t_{2}\right)-s\right)^{r-1} p\left(t_{1}, s\right) d_{s}\left(\bigvee_{0}^{s}\left[g\left(t_{2}, \cdot\right)-g\left(t_{1}, \cdot\right)\right]\right), \\
\left|I_{13}\right| \leq & \left.\int_{0}^{\beta\left(t_{1}\right)} \mid\left(\beta\left(t_{2}\right)-s\right)^{r-1}-\beta\left(t_{1}\right)-s\right)^{r-1} \mid p\left(t_{1}, s\right) d_{s}\left(\bigvee_{0}^{s} g\left(t_{2}, \cdot\right)\right),
\end{aligned}
$$

and, from the continuity of $\alpha, \beta, f, g, h$ and $p$, we see that the terms $I_{1}$ and $I_{2}$ tend to zero if $t_{1} \rightarrow t_{2}$, uniformly for $u \in B_{\eta}$.

Step 4. $N\left(B_{\eta}\right)$ is equiconvergent. Let $t \in \mathbb{R}_{+}$and $u \in B_{\eta}$. From (3.2), we have

$$
|(N u)(t)| \leq|f(t, 0,0)|+\frac{M \eta}{1+\alpha(t)}+L q(t),
$$

and thus $(N u)(t) \rightarrow 0=(N u)(+\infty)$ as $t \rightarrow+\infty$, uniformly for $u \in B_{\eta}$.

As a consequence of Steps 1-4, together with Lemma 2.7, we can conclude that $N: B_{\eta} \rightarrow B_{\eta}$ is continuous and compact. From an application of Schauder's theorem [16], we deduce that $N$ has a fixed point $u$ which is a solution of the equation (1.1).

Step 5. Global asymptotic stability of solutions. Now we investigate the stability of solutions to equation (1.1). Let us assume that $u$ and $v$ are two solutions of (1.1). Then, for each $t \in \mathbb{R}_{+}$, we have

$$
\begin{aligned}
|u(t)-v(t)| & =|(N u)(t)-(N v)(t)| \\
& =|f(t, u(\alpha(t)),(Q u)(t))-f(t, v(\alpha(t)),(Q u)(t))| \\
& \leq \frac{M|u(\alpha(t))-v(\alpha(t))|+L|(Q u)(t)-(Q v)(t)|}{1+\alpha(t)+\mid u(\alpha(t))-v(\alpha(t) \mid} \\
& \leq M|u(\alpha(t))-v(\alpha(t))|+L(|(Q u)(t)|+|(Q v)(t)|),
\end{aligned}
$$


and thus

$$
|u(t)-v(t)| \leq M|u(\alpha(t))-v(\alpha(t))|+2 L q(t) .
$$

Since $\alpha(t) \rightarrow \infty$ as $t \rightarrow \infty$,

$$
\lim _{t \rightarrow \infty}|u(\alpha(t))-v(\alpha(t))|=\lim _{t \rightarrow \infty}|u(t)-v(t)|,
$$

and thus, by using (3.4) and our assumption that $0<M<1$, we deduce that

$$
\lim _{t \rightarrow \infty}|u(t)-v(t)| \leq \lim _{t \rightarrow \infty} \frac{2 L q(t)}{1-M}=0 .
$$

Consequently, all solutions of equation (1.1) are globally asymptotically stable.

4. An example. As an application of our results we consider the following nonlinear, quadratic functional, Riemann-Liouville, VolterraStieltjes integral equation of fractional order,

$$
\begin{aligned}
u(t)=f(t, u(t), & \left.\frac{1}{\Gamma(2 / 3)} \int_{0}^{t}(t-s)^{-1 / 3} h(t, s, u(s)) d s\right) \\
& \text { for } t \in \mathbb{R}_{+},
\end{aligned}
$$

which is of the form (1.1) and (1.2) with $r=2 / 3, \alpha(t)=\beta(t)=\gamma(t)=t$ and $g(t, s)=s$ for $(t, s) \in \mathbb{R}_{+}^{2}$, and in which we put

$$
\begin{aligned}
& f(t, u, v)=\frac{1}{2} \frac{1}{|u|+2(1+t+|v|)}, \\
& h(t, s, u)=\frac{s|u|}{(1+t+|u|)\left(1+t^{4}\right)},
\end{aligned}
$$

for $(t, s) \in J_{1}:=\left\{(t, s) \in \mathbb{R}_{+}^{2}: s \leq t\right\}$ and $u, v \in \mathbb{R}$. For each $t \in \mathbb{R}_{+}$ and $u_{1}, u_{2}, v_{1}, v_{2} \in \mathbb{R},\left|f\left(t, u_{1}, v_{1}\right)-f\left(t, u_{2}, v_{2}\right)\right|$ is bounded by

$$
\begin{aligned}
& \frac{1}{2}\left|\frac{\left|u_{1}\right|-\left|u_{2}\right|+2\left|v_{1}\right|-2\left|v_{2}\right|}{\left(\left|u_{1}\right|+2+2 t+2\left|v_{1}\right|\right)\left(\left|u_{2}\right|+2+2 t+2\left|v_{2}\right|\right)}\right| \\
& \leq \frac{1}{2} \frac{|| u_{1}|-| u_{2}||+2|| v_{1}|-| v_{2}||}{\left(\left|u_{1}\right|+2+2 t+2\left|v_{1}\right|\right)\left(\left|u_{2}\right|+2+2 t+2\left|v_{2}\right|\right)} .
\end{aligned}
$$

Since

$$
|| u_{1}|-| u_{2}|| \leq\left|u_{1}-u_{2}\right| \leq\left|u_{1}\right|+\left|u_{2}\right|
$$


and

$$
\left(\left|u_{1}\right|+2+2 t+2\left|v_{1}\right|\right)\left(\left|u_{2}\right|+2+2 t+2\left|v_{2}\right|\right) \geq 1+t+\left|u_{1}\right|+\left|u_{2}\right|,
$$

we get

$$
\left|f\left(t, u_{1}, v_{1}\right)-f\left(t, u_{2}, v_{2}\right)\right| \leq \frac{(1 / 2)\left|u_{1}-u_{2}\right|+\left|v_{1}-v_{2}\right|}{1+t+\left|u_{1}-u_{2}\right|}
$$

so the function $f$ is continuous and satisfies assumption $\left(H_{1}\right)$, with $M=1 / 2$ and $L=1$. Also, $f$ satisfies assumption $\left(H_{2}\right)$ with $f^{*}=1 / 4$. Next, we can easily see that the function $g$ satisfies hypotheses $\left(H_{3}\right)_{-}$ $\left(H_{6}\right)$. Finally, the function $h$ satisfies assumption $\left(H_{7}\right)$, with

$$
p(t, s)=\frac{s}{1+t^{4}} \quad \text { for }(t, s) \in J_{1} .
$$

Indeed, we have,

$$
\begin{aligned}
\int_{0}^{\beta(t)}(\beta(t)-s)^{r-1} p(t, s) d_{s} g(t, s) & =\int_{0}^{t}(t-s)^{-1 / 3} \frac{s}{1+t^{4}} d s \\
& =\frac{9 t^{5 / 3}}{10\left(1+t^{4}\right)} \rightarrow 0 \text { as } t \rightarrow \infty
\end{aligned}
$$

Hence, by Theorem 3.3, equation (4.1) has a solution defined on $\mathbb{R}_{+}$, and all solutions of this equation are globally asymptotically stable.

Acknowledgments. The authors are grateful to the referees and the editor for their helpful remarks.

\section{REFERENCES}

1. S. Abbas and M. Benchohra, On the set of solutions of fractional order Riemann-Liouville integral inclusions, Demons. Math. 46 (2013), 271-281.

2. time delay, Appl. Math. E-Notes 12 (2012), 79-87.

3. - On the existence and local asymptotic stability of solutions of fractional order integral equations, Comm. Math. 52 (2012), 91-100.

4. S. Abbas, M. Benchohra and J. Henderson, On global asymptotic stability of solutions of nonlinear quadratic Volterra integral equations of fractional order, Comm. Appl. Nonlin. Anal. 19 (2012), 79-89.

5. S. Abbas, M. Benchohra and G.M. N'Guérékata, Topics in fractional differential equations, Springer, New York, 2012.

6. , Advanced fractional differential and integral equations, Nova Science Publishers, New York, 2015. 
7. S. Abbas, M. Benchohra, M. Rivero and J. Trujillo, Existence and stability results for nonlinear fractional order Riemann-Liouville Volterra-Stieltjes quadratic integral equations, Appl. Math. Comp. 247 (2014), 319-328.

8. S. Abbas, M. Benchohra and A.N. Vityuk, On fractional order derivatives and Darboux problem for implicit differential equations, Frac. Calc. Appl. Anal. 15 (2012), 168-182.

9. J. Appell, J. Banas and N. Merentes, Variation and around, Ser. Nonlin. Anal. Appl. 17, Walter de Gruyter, Berlin, 2014.

10. D. Baleanu, K. Diethelm, E. Scalas and J.J. Trujillo, Fractional calculus models and numerical methods, World Scientific Publishing, New York, 2012.

11. J. Banas̀ and K. Goebel, Measures of noncompactness in Banach spaces, Marcel Dekker, New York, 1980.

12. J. Banaś and B. Rzepka, On existence and asymptotic stability of solutions of a nonlinear integral equation, J. Math. Anal. Appl. 284 (2003), 165-173.

13. , Monotonic solutions of a quadratic integral equation of fractional order, J. Math. Anal. Appl. 332 (2007), 1371-1379.

14. C. Corduneanu, Integral equations and stability of feedback systems, Academic Press, New York, 1973.

15. M.A. Darwish, J. Henderson and D. O'Regan, Existence and asymptotic stability of solutions of a perturbed fractional functional integral equations with linear modification of the argument, Bull. Kor. Math. Soc. 48 (2011), 539-553.

16. A. Granas and J. Dugundji, Fixed point theory, Springer-Verlag, New York, 2003.

17. R. Hilfer, Applications of fractional calculus in physics, World Scientific, Singapore, 2000.

18. A.A. Kilbas, H.M. Srivastava and J.J. Trujillo, Theory and applications of fractional differential equations, Elsevier Science B.V., Amsterdam, 2006.

19. V. Lakshmikantham, S. Leela and J. Vasundhara, Theory of fractional dynamic systems, Cambridge Academic Publishers, Cambridge, 2009.

20. K.S. Miller and B. Ross, An Introduction to the fractional calculus and differential equations, John Wiley, New York, 1993.

21. I.P. Natanson, Theory of functions of a real variable, Ungar, New York, 1960.

22. I. Podlubny, Fractional differential equations, Academic Press, San Diego, 1999.

23. R. Sikorski, Real functions, PWN, Warsaw, 1958 (in Polish).

24. V.E. Tarasov, Fractional dynamics. Applications of fractional calculus to dynamics of particles, fields and media, Springer, Heidelberg, 2010.

25. Y. Zhou, Basic theory of fractional differential equations, World Scientific, Singapore, 2014. 
Laboratory of Mathematics, University of SaÏDA, P.O. Box 138, 20000 SAÏDA, Algeria

Email address: abbasmsaid@yahoo.fr

Laboratory of Mathematics, University of Sidi Bel-Abbès, P.O. Box 89, Sidi Bel Abbes 22000, Algeria and Department of Mathematics, Faculty of Science, King Abdulaziz University, P.O. Box 80203, Jeddah 21589, Saudi Arabia

Email address: benchohra@univ-sba.dz, benchohra@yahoo.com

Faculté des Sciences de l’Ingénieur, Université de Tlemcen, B.P. 119, 13000 , Tlemcen, Algérie

Email address: ba_slimani@yahoo.fr

Departamento de Análisis Matemático, Universidad de La Laguna, 38271 La Laguna, Tenerife, Spain

Email address: jtrujill@ullmat.es 